\title{
A hidden constraint on the Hamiltonian formulation of relativistic worldlines
}

\author{
Benjamin Koch ${ }^{\text {a }}$, Enrique Muñoz \\ Pontificia Universidad Católica de Chile, Instituto de Física, Pontificia Universidad Católica de Chile, Casilla 306, Santiago, Chile
}

Received: 4 February 2019 / Accepted: 5 November 2019 / Published online: 19 November 2019

(C) The Author(s) 2019

\begin{abstract}
Gauge theories with general covariance are particularly reluctant to quantization. We discuss the example of the Hamiltonian formulation of the relativistic point particle that, despite its apparent simplicity, is of crucial importance since a number of point particle systems can be cast into this form on a higher dimensional Rindler background, as recently pointed out by Hojman. It is shown that this system can be equipped with a hidden local, symmetry generating, constraint which on the one hand does not bother the classical evolution and on the other hand simplifies the realization of the path integral quantization. Even though the positive impact of the hidden symmetry is more evident in the Lagrangian version of the theory, it is still present through the suggested Hamiltonian constraint.
\end{abstract}

\section{Contents}

1 Introduction ................. 1

2 Hamiltonian PI for the RPP . . . . . . . . . . 2

3 Discussion summary and outlook ........ 5

References............... 5

\section{Introduction}

Symmetries have been the guiding principle of theoretical physics throughout centuries. In particular local symmetries have shown to be particularly useful for the description of fundamental interactions. The description of all four known forces of nature: electromagnetism, weak interaction, strong interaction, and gravitation have been cast in this language. However, the last member of this illustrious list poses serious problems, when it comes to a quantum formulation of gravity. Numerous attempts have been made to solve the problem but, up to now, no conclusion could be reached (see $[1,2]$

\footnotetext{
a e-mail: bkoch@fis.puc.cl
}

for a review). Clearly, one needs to better understand the quantization of this theory, based on a particularly beautiful and complicated symmetry called general covariance. Since the rich structure of the full gravitational covariant system appears to be too complicated to tackle the problem directly, it seems instead that a wiser strategy is to learn more about general covariance in simpler systems.

Probably, the simplest system with general covariance is the relativistic point particle (RPP) [3-8]. Even though the quantization of the RPP was achieved in the Hamiltonian version of this theory, attempts to quantize the Lagrangian version of the action of the relativistic point particle

$S=\int d \lambda \sqrt{\frac{d x^{\mu}}{d \lambda} \frac{d x_{\mu}}{d \lambda}}$

lead to deep problems and inconsistencies. A Lagrangian action is most naturally quantized in a path integral (PI) approach, so in what follows we shall refer to this quantization method. The attitudes towards this problem that can be found in the literature are:

(a) declare the Lagrangian action (1) to be wrong or at least inadequate for the purpose of quantization, and stick to the Hamiltonian version. This Hamiltonian version of the action was first formulated in $[6,9,10]$;

(b) stick to the straight forward PI quantization of (1) and try to tackle the arising problems by a re-definition of the usual interpretation of probability, the super-probability $[11,12]$;

(c) study the system on special manifolds [13] or use approximations [7,8];

(d) realize that the action has a hidden symmetry, which, when factored out of the PI solves the inconsistencies and the quantization works just fine, giving the expected results. The factorization was shown to work in a formal Fadeev Popov construction [14] and in a purely geometrical approach [15]. 
Clearly the option (d) is the most favorable solution, due to its straightforward conceptual interpretation and its effectiveness at yielding the correct answers. The key point of this solution was that it treated two paths which are connected by the symmetry of local velocity rotations as physically equivalent. Geometrically this symmetry transformation will turn out to deform virtual paths orthogonal to the instantaneous momentum under the condition to keep the total action $S>S_{c l}$ of this path constant. Factoring out those paths from the naive PI calculation solved the aforementioned pathologies. When one tries to connect this astonishing result with the PI quantization of the corresponding Hamiltonian system, several questions arise:

Is there a corresponding additional symmetry in the Hamiltonian system? If it exists, can this additional symmetry be written in terms of a (local) constraint? If this can be done, how does this constrain affect the PI construction of the Hamiltonian system?

On the following pages, those questions will be answered. The paper is organized as follows: We first start with a thorough discussion of the different criteria that a Hamiltonian formulation of the path integral for the RPP must meet, in order to capture the symmetries involved. Then, we discuss the consequences of treating a Hamiltonian formulation that does not take into account the hidden symmetry. Finally, we propose an ansatz to involve this symmetry explicitly in the action, via a suitable constraint and a corresponding Lagrange multiplier. Moreover, by explicit calculation of the path integral, we show that this constraint does indeed allow us to recover the correct result for the RPP propagator. At last, we present the conclusions and possible extensions of our formulation to other physical systems of current interest.

\section{Hamiltonian PI for the RPP}

The aim is to formulate a Hamiltionian theory for the relativistic point particle that meets several criteria.

Let us summarize those in the following table, where the central column describes the criteria and the right column the motivation.

\subsection{Hamiltonian without hidden symmetry: summary}

The usual Hamiltonian action of the RPP is

$S[x, p, n]=\int_{t_{1}}^{t_{f}} d t \quad\left[\dot{x} \cdot p-n\left(p^{2}-m^{2}\right)\right]$,

where $x^{\mu}$ is the position variable, $p^{\mu}$ is the momentum variable, and $n$ is the Lagrange multiplier imposing the Hamiltonian constraint

$H_{0}=\phi=p^{2}-m^{2}$.
Let's summarize the most important properties of this action. It is invariant under global transformations $x^{\mu} \rightarrow x^{\mu}+\xi^{\mu}$, where $\xi^{\mu}$ is a constant. It is further invariant under the local transformations generated by (3)

$\delta x^{\mu}(\lambda)=\left\{x^{\mu}(\lambda), \int d \lambda^{\prime} \epsilon\left(\lambda^{\prime}\right) \phi\left(\lambda^{\prime}\right)\right\}=2 \epsilon(\lambda) p^{\mu}(\lambda)$,

where the canonical Poisson bracket $\left\{x^{\mu}(\lambda), p_{\nu}\left(\lambda^{\prime}\right)\right\}=$ $\delta_{v}^{\mu} \delta\left(\lambda-\lambda^{\prime}\right)$ was used. The classical equations of motion for (2) are

$$
\begin{aligned}
\dot{p}^{\mu} & =0, \\
\dot{x}^{\mu} & =2 n p^{\mu}, \\
p^{2}-m^{2} & =0 .
\end{aligned}
$$

The path integral over (2) that defines the propagator

$$
\left\langle x_{f}^{\mu}-x_{1}^{\mu}\right\rangle=\int_{x\left(t_{1}\right)=x_{1}}^{x\left(t_{f}\right)=x_{f}} \mathcal{D}[x(t)] \mathcal{D}[p(t)] \mathcal{D}[n(t)] e^{i S[x, p, n]}
$$

can be obtained from a straight forward calculation. Time discretization is performed as usual over $F$-intervals, such that $\epsilon=\left(t_{f}-t_{1}\right) / F$ is the size of each time-slice. Hence, for $t_{j}=t_{1}+(j-1) \epsilon,(1 \leq j \leq F)$, the discrete coordinates $x\left(t_{j}\right) \rightarrow x_{j}$, and momenta $p\left(t_{j}\right) \rightarrow p_{j}$, with fixed coordinates at the ends $x\left(t_{1}\right) \rightarrow x_{1}$ and $x\left(t_{f}\right) \rightarrow x_{f}$, respectively.

Similarly, the integration measure over paths becomes, in the discretised form

$\mathcal{D}[x(t)] \mathcal{D}[p(t)] \mathcal{D}[n(t)] \rightarrow \prod_{j=2}^{F-1} d^{d} x_{j} \prod_{j=1}^{F} d^{d} p_{j} d n_{j}$.

For example, one can perform the $\int d^{d} x_{j}$ integrals first, which give delta functions in momenta, of the form $\delta^{d}\left(p_{j+1}^{\mu}-\right.$ $p_{j}^{\mu}$ ). These delta functions allow to perform all the momenta integrals $\int d^{d} p_{j}$, except for the final one. The integrals over the Lagrange multipliers of the constraint $\int d n_{j}$, would give a delta function $\delta\left(p_{j}^{2}-m^{2}\right)$ each. In order to avoid this piling up of delta functions, one fixes all but one $n_{j}$, thus giving the expected propagator

$$
\left\langle x_{f}^{\mu}-x_{1}^{\mu}\right\rangle=\mathcal{N} \int d^{d} p_{F} e^{-i\left(x_{f}-x_{1}\right) \cdot p_{F}} \delta\left(p_{F}^{2}-m^{2}\right) .
$$

\subsection{Ansatz for the constraint}

As listed above, one wants a constraint which reflects the velocity rotations in the Lagrangian picture. Since velocity is a vector, a scalar constraint is insufficient. One needs at least a vector or tensor for this task. Further, in the equations of motion (5-7) the velocity is associated to the momentum, thus one might first attempt to formulate a constraint which transforms the momenta $p^{\mu}$. In order to transform the momenta with a Poisson bracket with $\phi^{\mu}$, one needs this 
Table 1 Summary of different criteria and corresponding motivation for symmetry-related constraints in the Hamiltonian formulation

\begin{tabular}{|c|c|c|}
\hline & Criterium & Motivation \\
\hline (1) & $\begin{array}{l}\text { The Hamiltonian action includes an additional constraint } \phi^{\mu} \\
\text { that reflects the local velocity rotations symmetry discussed } \\
\text { in the Lagrangian formulation }\end{array}$ & $\begin{array}{l}\text { This is imposed because the original motivation is to find the } \\
\text { meaning of the local velocity rotations in the Hamiltonian } \\
\text { picture }\end{array}$ \\
\hline (2) & $\begin{array}{l}\text { The constraint } \phi^{\mu} \text { generates local symmetry transformation of } \\
\text { the action }\end{array}$ & $\begin{array}{l}\text { A local symmetry is needed in order to justify a later } \\
\text { factorization from the PI, similar to the known redundant } \\
\text { gauge configurations }\end{array}$ \\
\hline (3) & $\begin{array}{l}\text { The equations of motion are in agreement with the classical } \\
\text { equations of the RPP }\end{array}$ & $\begin{array}{l}\text { Only if the systems are classically equivalent, one is still } \\
\text { solving the same problem one was up for in the first place, } \\
\text { namely the quantization of the RPP }\end{array}$ \\
\hline (4) & $\begin{array}{l}\text { The PI of the additional constraint can be done and the result } \\
\text { does not modify the expected Klein Gordon propagator }\end{array}$ & $\begin{array}{l}\text { The factorization of the new symmetry is meant to act as } \\
\text { improvement of the naive PI approach and thus should not } \\
\text { introduce modifications where this naive approach already } \\
\text { works }\end{array}$ \\
\hline
\end{tabular}

constraint to depend on the positions $\phi^{\mu}=\phi^{\mu}(x)$. However, when working out the equations of motion and algebra, it becomes clear that such a position dependent constraint would have to be non-local in the position variables. This can be done, but we prefer to avoid the problems that come along with non-locality, and hence we search for a constraint that is local in momentum space but independent of $x^{\nu}$, namely $\phi^{\mu}=\phi^{\mu}(p)$. Still a change in the momentum can be achieved but in a different way, as will be seen.

The action with the new constraint is then

$S[x, p, n, N]=\int_{t_{1}}^{t_{f}} d t \quad\left[\dot{x} \cdot p-n\left(p^{2}-m^{2}\right)-N_{\mu} \phi^{\mu}\right]$.

Here, we propose

$$
\begin{aligned}
\phi^{\mu} & =\dot{x}^{\mu}\left(\frac{\delta H_{0}}{\delta p}\right)^{2}-\left(\frac{\delta H_{0}}{\delta p}\right)^{\mu}\left(\dot{x} \cdot\left(\frac{\delta H_{0}}{\delta p}\right)\right) \\
& =\dot{x}^{\mu} p^{2}-p^{\mu}(\dot{x} \cdot p),
\end{aligned}
$$

where $N^{\mu}$ is the new Lagrange multiplier function that must vanish at the endpoints and $H_{0}=\phi$ is the usual Hamiltonian constraint.

Notice that the second constraint term in the action Eq. (11) can also be written

$N_{\mu} \phi^{\mu}=\dot{x}_{\mu} \hat{\phi}^{\mu}$,

where we have defined

$\hat{\phi}^{\mu}=N^{\mu} p^{2}-p^{\mu}(N \cdot p)$.

This second form of the constraint has the useful property that it is by construction orthogonal to the momentum, namely

$p_{\mu} \hat{\phi}^{\mu}=0$,

which will lead to very useful simplifications as discussed in the next section.

\subsection{Equations of motion}

One can derive the equations of motion for this system in the variables $x^{\mu}, p^{\mu}$, and $N^{\mu}$. Then, we find that $N^{\mu} \sim p^{\mu}$ and thus that the equations of motion are equivalent to the usual ones. ${ }^{1}$ This can be seen more elegantly if one notes that $p^{\mu}$ is not the canonical momentum $\pi^{\mu}$ any more since introducing (14) gives

$\pi^{\mu}=p^{\mu}-\hat{\phi}^{\mu}$.

This is the change in the momentum variable we have been seeking. Thus, in order to derive the equations of motion for the system with $\pi^{\mu}$, one would like to rewrite the whole action in terms of $\pi^{\mu}$ instead of $p^{\mu}$. By using (15) and (16), one finds that

$p^{2}=\pi^{2}-\hat{\phi}^{2}=\pi^{2}-p^{2} N_{\mu} \hat{\phi}^{\mu}$.

Let us now reformulate the constraint Eq. (14) in terms of the new canonical momenta $\pi^{\mu}$, by considering the expression in the action:

$$
\begin{aligned}
N_{\mu} \hat{\phi}^{\mu}= & N^{2} p^{2}-(N \cdot p)^{2} \\
= & N_{\mu}\left[N^{\mu} \pi^{2}-(N \cdot \pi) \pi^{\mu}\right] \\
& -\hat{\phi}^{\mu}\left[N^{2} \hat{\phi}_{\mu}-N_{\mu}(N \cdot \hat{\phi})-2(N \cdot \pi) N_{\mu}\right] .
\end{aligned}
$$

While the first term represents the original constraint expressed in terms of the new canonical momenta, the second term is clearly proportional to $\hat{\phi}^{\mu}$ and can be reabsorbed into thedefinition of the Lagrange multiplier $N^{\mu}$ by a trivial

\footnotetext{
1 It is important to realize that this classical reduction to the usual equations of motion fails for the non-relativistic theory, because in this theory the classical relation between $\dot{\mathbf{x}}$ and $\mathbf{p}$ is fixed and an arbitary $\mathbf{N}$ which is just proportional to $\mathbf{p}$ would break this relation.
} 
shift. Therefore, after shifting and rescaling $N^{\mu} \rightarrow \tilde{N}^{\mu}=$ $N^{\mu} \sqrt{n p^{2}}$, the action (11) can be written as

$S[x, \pi, n, \tilde{N}]=\int_{t_{1}}^{t_{f}} d t\left[\dot{x} \cdot \pi-n\left(\pi^{2}-m^{2}\right)-\tilde{N} \cdot \tilde{\phi}\right]$,

where

$\tilde{\phi}^{\mu}=\tilde{N}^{\mu} \pi^{2}-\pi^{\mu}(\tilde{N} \cdot \pi)$.

After a variation with respect to $\delta x^{\mu}, \delta \pi^{\mu}, \delta n$, and $\delta \tilde{N}^{\mu}$, the equations of motion are respectively

$$
\begin{aligned}
\dot{\pi}^{\mu} & =0, \\
\dot{x}^{\mu} & =2 n \pi^{\mu}+2\left(\pi^{\mu} \tilde{N}^{2}-\tilde{N}^{\mu}(\tilde{N} \cdot \pi)\right), \\
\pi^{2}-m^{2} & =0, \\
\tilde{\phi}^{\mu} & =0=\tilde{N}^{\mu} \pi^{2}-\pi^{\mu}(\tilde{N} \cdot \pi) .
\end{aligned}
$$

Note that, in order to compare this with (5-7), one should now relabel $\pi^{\mu} \rightarrow p^{\mu}$. One notes then, that the equations (5) and (7) are unchanged with respect to (21) and (23). The equation (22) acquired an additional term with respect to (6). However, this modification vanishes on-shell due to the new equation (24), which forces $\tilde{N}^{\mu}$ to be parallel to $\tilde{\phi}^{\mu}$. Thus, the extended action (19) is equivalent to the action (2) at the classical level. Also all classical symmetries of (2) are present in (19), just as required.

We remark that the introduction of the new constraint $\tilde{\phi}^{\mu}$, Eq.(20) does not affect the classical dynamics of the relativistic point particle, since after Eqs. (23) and (24), we solve

$\tilde{N}^{\mu}=\frac{\tilde{N} \cdot \pi}{m^{2}} \pi^{\mu}$.

Substituted into Eq. (23), yields

$\dot{x}^{\mu}=2 n \pi^{\mu}$,

that leaves the usual time-reparametrization $\lambda=2 n x^{0}$ as the only degree of freedom to be fixed, to recover the usual classical dynamics

$\frac{d x^{\mu}}{d \lambda}=\pi^{\mu}, \quad \frac{d^{2} x^{\mu}}{d \lambda^{2}}=\frac{d \pi^{\mu}}{d \lambda}=0$.

\subsection{The algebra of local transformations}

The term $\tilde{\phi}=\tilde{N}_{\mu} \cdot \tilde{\phi}^{\mu}$ generates local transformations. The algebra (with $\partial / \partial \pi^{i}$ instead of $\partial / \partial p^{i}$ ) is simply

$\left\{\tilde{\phi}(t), \tilde{\phi}\left(t^{\prime}\right)\right\}=0$.

Canonical momenta are unchanged under this constraint

$\left\{\pi^{\mu}(t), \tilde{\phi}\left(t^{\prime}\right)\right\}=0$, but it generates a local transformation of the position variables

$$
\delta x^{\mu}=\left\{x^{\mu}(t), \int_{t_{1}}^{t_{f}} d t^{\prime} \tilde{\phi}\left(t^{\prime}\right)\right\}=2\left(\tilde{N}^{2} \pi^{\mu}-(\tilde{N} \cdot \pi) N^{\mu}\right) .
$$

One realizes that this transformation is orthogonal to the instantaneous momentum direction

$\delta x \cdot \pi=0$.

Thus, $\delta x^{\mu}$ reflects the spirit of the velocity rotations in the Lagrangian formulation $[14,15]$. The constraint further generates a variation of the action

$$
\begin{aligned}
\delta_{\tilde{\phi}} S= & \int_{t_{1}}^{t_{f}} d t[\delta(\dot{x} \cdot \pi)-\delta \tilde{\phi}] \\
= & \int_{t_{1}}^{t_{f}} d t\left[\frac{d}{d t}\left(\tilde{N}^{2} \pi^{2}-(\tilde{N} \cdot \pi)^{2}\right)\right. \\
& \left.-2(\tilde{N} \cdot(\dot{\tilde{N}}+\delta \tilde{N})) \pi^{2}+2(\tilde{N} \cdot \pi)((\dot{\tilde{N}}+\delta \tilde{N}) \cdot \pi)\right] .
\end{aligned}
$$

This variation reduces to a boundary term if the Lagrange multiplier transforms as

$\delta \tilde{N}^{\mu}=-\dot{\tilde{N}}^{\mu}$.

Thus, $\tilde{\phi}$ generates a local symmetry of the action $S$.

\subsection{Path integral}

For the PI one uses $N^{\mu}$ without rescaling, which in the discretized version reads

$$
\begin{aligned}
S\left[x^{\mu}, \pi^{\nu}, n, N^{\alpha}\right]= & \sum_{i=1}^{F} \epsilon\left(\left(\pi_{i} \cdot\left(x_{i+1}-x_{i}\right)\right) / \epsilon\right. \\
& \left.-n_{i}\left(\pi_{i}^{2}-m^{2}\right)-n_{i} \pi_{i}^{2}\left(N_{i} \cdot \phi_{i}\right)\right) .
\end{aligned}
$$

The functional integrals are

$$
\begin{aligned}
& \int \mathcal{D} x=\Pi_{i=2}^{F-1} \int d^{d} x_{i} \\
& \int \mathcal{D} \pi=\Pi_{i=1}^{F} \int d^{d} \pi_{i} \\
& \int \mathcal{D} n=\Pi_{i=1}^{F} \int d n_{i} \\
& \int \mathcal{D} N=\Pi_{i=1}^{F-1} \int d^{d-1} N_{o, i} \cdot \Omega_{i} .
\end{aligned}
$$

Here, we have split the vectors $N_{i}^{\mu}$ into a part orthogonal to $\pi_{i}^{\mu}$ and a part parallel to $\pi_{i}^{\mu}$

$N_{i}^{\mu}=N_{o, i}^{\mu}+N_{i, \|}^{\mu}$. 
Clearly, in the PI one should only integrate over the orthogonal part $N_{o, i}^{\mu}$, since the parallel part does not contribute to the action (19). The properly chosen measure for this integration $\int d^{d-1} N_{o, i}$ is $\Omega_{i}$. Note that this adjustment of the measure is not unusual, since it is known that canonical transformations, similar to those generated by $N_{o, i}^{\mu}$, can result in a change of the path integral measure [16-18].

As mentioned after (11) we further impose that the final $N_{o, F}^{\mu}=0$. Let us perform the integrals (35-38) blockwise for each time step. The first block contains $\int d^{d} x_{2} \int d^{d} \pi_{1} \int d n_{1} \int d^{d-1} N_{1} \Omega_{1}$ which will now be integrated in the same order

$$
\begin{aligned}
\left\langle x_{f}^{\mu}-x_{1}^{\mu}\right\rangle= & \left(\int d^{d} x_{2} \int d^{d} \pi_{1} \int d n_{1} \int d^{d-1} N_{o, 1}\right) \\
& \cdot e^{i\left[\left(\left(x_{2}-x_{1}\right) \cdot\left(\pi_{2}-\pi_{1}\right)\right)-\epsilon n_{1}\left(\pi_{1}^{2}\left(1+N_{1}^{2}\right)-m^{2}\right)\right]} \ldots
\end{aligned}
$$

Now, integrating over $\int d^{d} x_{2}$ gives a $\delta$-function in $\pi^{\mu}$, which can be used to integrate $\int d^{d} \pi_{1}$. Then integrating in $\int d n_{1}$ gives another $\delta$-function

$\delta\left(\pi_{2}^{2}\left(1+N_{o, 1}^{2}\right)-m^{2}\right)$,

which can be used to evaluate the "radial" part of the $\int d^{d-1} N_{o, 1}$ integral (the angular part is just normalization). For a proper choice of the measure $\Omega_{1}$, all integrals in this set have canceled each other and the contribution is just a multiplicative one. This procedure continues until the final integrals, which have no $\int d^{d} x_{F}$ and no $\int d^{d-1} N_{o, F}$, and which thus read

$$
\begin{aligned}
\left\langle x_{f}^{\mu}-x_{1}^{\mu}\right\rangle & =\mathcal{N}^{\prime} \cdot \int d^{d} \pi_{F} \int d n_{F} e^{-i\left(\left(x_{f}-x_{1}\right) \cdot p_{F}-\epsilon n_{F}\left(\pi_{F}^{2}-m^{2}\right)\right)} \\
& =\mathcal{N} \int d^{d} \pi_{F} e^{-i\left(\left(x_{f}-x_{1}\right) \cdot \pi_{F}\right)} \delta\left(\pi_{F}^{2}-m^{2}\right)
\end{aligned}
$$

where $\mathcal{N}^{\prime}$ and $\mathcal{N}$ are just normalization constants. This is the desired usual result (10).

It is very interesting to note, that in this PI construction the integrals of the two constraints canceled each other and thus, we did not even have to fix some gauges explicitly as in the usual case. This non-trivial impact on the PI formulation gives further evidence, that the new constraint, even though "hidden" at the classical level, is not "trivial" at the level of the quantum mechanical path integral formulation.

\section{Discussion summary and outlook}

We have presented a new constraint (12) for the RPP and shown that it reflects all the nice features demanded in table 1 . In particular, it generates a non-trivial local symmetry of the action without altering the classical equations of motion of the system. This new PI formulation worked out in a straight forward way. Based on our results, we can conclude that the local symmetry of the Lagrangian version of the RPP action (1), which was discussed in $[14,15]$ can also be implement in the Hamiltonian version (19).

In the discussion of constraints, it is a useful exercise to count the degrees of freedom. For the symmetry presented in this paper, this discussion has to be differentiated between classical paths $S=S_{c l}$ and quantum paths $S>S_{c l}$. For the former it has been shown that the additional constraints are ineffective, leaving the usual degrees of freedom of a relativistic point particle. For the latter, however, the constraints become effective. In this case, three of the four conditions are independent, reducing the degrees of freedom for a given action $S$ to zero. As shown in Sect. 2.5, this leaves, after applying the constraints, only one integral corresponding to $\int d S$ itself for the path integral.

This seems to be a very isolated result, only valid for a very particular system with general covariance. However, as recently shown by Hojman, there is a very large class of point particle systems which can be cast in the form of the free RPP which is living on a higher dimensional Rindler background $[19,20]$. Thus, we believe that our results might be applicable to a much larger class of problems. Further, it would be interesting to explore certain similarities of the presented constraint with constraints imposed in delta-theories such as delta gravity [21]. Finally, our result encourages further investigation on more complicated covariant systems such as quantum cosmology [22] or ultimately quantum gravity in the canonical formulation [23] analogous to (2/19).

Acknowledgements B. K. was supported by Fondecyt 1161150 and Fondecyt 1181694. E. M. was supported by Fondecyt Regular 1190361.

Data Availability Statement This manuscript has no associated data or the data will not be deposited. [Authors' comment: This is a theoretical article and no experimental data has been listed.]

Open Access This article is distributed under the terms of the Creative Commons Attribution 4.0 International License (http://creativecomm ons.org/licenses/by/4.0/), which permits unrestricted use, distribution, and reproduction in any medium, provided you give appropriate credit to the original author(s) and the source, provide a link to the Creative Commons license, and indicate if changes were made. Funded by $\mathrm{SCOAP}^{3}$.

\section{References}

1. C. Kiefer, Annalen Phys. 15, 129 (2005). https://doi.org/10.1002/ andp. 200510175

2. C. Kiefer, Ann. Phys. 518, 129 (2006). https://doi.org/10.1002/ andp. 200510175. arXiv:gr-qc/0508120

3. C. Teitelboim, Phys. Rev. D 25, 12 (1982)

4. M. Henneaux, C. Teitelboim, Ann. Phys. 143, 127 (1982). https:// doi.org/10.1016/0003-4916(82)90216-0 
5. I.H. Redmount, W.M. Suen, Int. J. Mod. Phys. A 8, 1629 (1993). https://doi.org/10.1142/S0217751X93000667. arXiv:gr-qc/9210019

6. E.S. Fradkin, D.M. Gitman, Phys. Rev. D 44, 3230 (1991). https:// doi.org/10.1103/PhysRevD.44.3230

7. T. Padmanabhan, Foundations of Physics 25, 11 (1994). https:// doi.org/10.1007/BF02054782

8. T. Padmanabhan, Found Phys 24, 1543 (1994). https://doi.org/10. 1007/BF02054782

9. L. Brink, S. Deser, B. Zumino, P. Di Vecchia, P.S. Howe, Phys. Lett. B 64, 435 (1976)

10. L. Brink, P. Di Vecchia, P.S. Howe, Nucl. Phys. B 118, 76 (1977)

11. P. Jizba, H. Kleinert, Phys. Rev. E 78, 031122 (2008). https://doi. org/10.1103/PhysRevE.78.031122

12. P. Jizba, H. Kleinert, Phys. Rev. D 82, 085016 (2010). https://doi. org/10.1103/PhysRevD.82.085016. arXiv:1007.3922 [hep-th]

13. H. Fukutaka, T. Kashiwa, Annals Phys. 176, 301 (1987). https:// doi.org/10.1016/0003-4916(87)90004-2

14. B. Koch, E. Muñoz, I. Reyes, Phys. Rev. D 96(8), 085011 (2017). https://doi.org/10.1103/PhysRevD.96.085011. arXiv:1706.05386 [hep-th]
15. B. Koch, E. Muñoz, Eur. Phys. J. C 78(4), 278 (2018). https://doi. org/10.1140/epjc/s10052-018-5753-9. arXiv:1706.05388 [hep-th]

16. M.S. Swanson, Phys. Rev. A 50, 4538 (1994). https://doi.org/10. 1103/PhysRevA.50.4538. arXiv:hep-th/9406167

17. M. Blasone, P. Jizba, L. Smaldone, Annals Phys. 383, 207 (2017). https://doi.org/10.1016/j.aop.2017.05.022. arXiv:1703.04314 [hep-th]

18. M. Blasone, P. Jizba, L. Smaldone, J. Phys. Conf. Ser. 804(1), 012006 (2017). https://doi.org/10.1088/1742-6596/804/1/012006

19. S.A. Hojman, Phys. Rev. D 98(8), 084037 (2018). https://doi.org/ 10.1103/PhysRevD.98.084037

20. S. A. Hojman. arXiv:1812.01974 [physics.gen-ph]

21. J. Alfaro, Phys. Part. Nucl. 44, 175 (2013). https://doi.org/10.1134/ S1063779613020032

22. A. Vilenkin, Phys. Rev. D 50, 2581 (1994). https://doi.org/10.1103/ PhysRevD.50.2581. arXiv:gr-qc/9403010

23. R.L. Arnowitt, S. Deser, C.W. Misner, Phys. Rev. 117, 1595 (1960). https://doi.org/10.1103/PhysRev.117.1595 